International Journal of Modern Physics A

(C) World Scientific Publishing Company

\title{
COMPARISON BETWEEN EXPERIMENT AND THEORY FOR THE THERMAL CASIMIR FORCE
}

\author{
G. L. KLIMCHITSKAYA \\ North-West Technical University, Millionnaya Street 5, St.Petersburg, 191065, Russia \\ Galina.Klimchitskaya@itp.uni-leipzig.de \\ M. BORDAG \\ Institute for Theoretical Physics, Leipzig University, Postfach 100920, D-04009, Leipzig, \\ Germany \\ V. M. MOSTEPANENKO \\ Noncommercial Partnership "Scientific Instruments", Tverskaya Street 11, Moscow, 103905, \\ Russia \\ Received 17 November 2011 \\ Revised 14 January 2012
}

\begin{abstract}
We analyze recent experiments on measuring the thermal Casimir force with account of possible background effects. Special attention is paid to the validity of the proximity force approximation (PFA) used in the comparison between the experimental data and computational results in experiments employing a sphere-plate geometry. The PFA results are compared with the exact results where they are available. The possibility to use fitting procedures in theory-experiment comparison is discussed. On this basis we reconsider experiments exploiting spherical lenses of centimeter-size radii.
\end{abstract}

Keywords: Thermal Casimir force; Drude model; precise measurements.

PACS numbers: 12.20.-m, 12.20.Fv, 78.20.Ci

\section{Introduction}

During the last ten years a lot of attention was devoted to measurements of the Casimir force 1 and to comparison between the experimental data and theoretical predictions. ${ }^{2-8}$ It is well known that predictions of the Lifshitz theory for the thermal Casimir force strongly depend on models of the dielectric permittivity used in computations. Typically the dielectric permittivity of ideal dielectric materials (i.e., isolators of infinitely high resistivity) is determined by the core electrons and can be presented in an oscillator form

$$
\varepsilon_{c}(\omega)=1+\sum_{j=1}^{K} \frac{g_{j}}{\omega_{j}^{2}-\omega^{2}-i \gamma_{j} \omega},
$$


where $K$ is the number of oscillators, $\omega_{j}$ are the oscillator frequencies, $g_{j}$ are the oscillator strengths, and $\gamma_{j}$ are the damping parameters. Real dielectrics, however, at any nonzero temperature possess some static dc conductivity $\sigma_{0}(T)$. As a result, their dielectric permittivity is given by

$$
\varepsilon_{d}(\omega)=\varepsilon_{c}(\omega)+i \frac{4 \pi \sigma_{0}(T)}{\omega} .
$$

For real metals rather good representation for the dielectric permittivity is given by the Drude model

$$
\varepsilon_{D}(\omega)=\varepsilon_{c}(\omega)-\frac{\omega_{p}^{2}}{\omega[\omega+i \gamma(T)]},
$$

where $\omega_{p}$ is the plasma frequency and $\gamma(T)$ is the relaxation parameter. In the region of infrared frequencies it holds $\gamma(T) \ll \omega$ and (3) converts to the so-called plasma model

$$
\varepsilon_{p}(\omega)=\varepsilon_{c}(\omega)-\frac{\omega_{p}^{2}}{\omega^{2}}
$$

This model disregards relaxation which does not play any role at so high frequencies. It was proved $\sqrt[5|6| 9 \mid 10]{ }$ that the Lifshitz theory combined with the most realistic permittivities (2) and (3) violates the third law of thermodynamics (the Nernst heat theorem) for dielectrics and metals with perfect crystal lattices, respectively. This result was originally proven for the configuration of two plane-parallel plates. In Ref. 11 the violation of the Nernst heat theorem for metals with perfect crystal lattices described by the Drude model and for dielectrics with included dc conductivity was demonstrated for the configuration of a sphere above a plate. This makes particularly important the experimental confirmation of one or other model of dielectric permittivity mentioned above.

In this paper we analyze several recent experiments which are sufficiently exact to discriminate between theoretical results computed using different models for the dielectric permittivity. We discuss the validity of the approximations employed to compare experiment with theory and the role of some background effects, such as patch potentials. Section 2 is devoted to the accuracy of the proximity force approximation (PFA). In Sec. 3 we consider the experimental results $12 \mid 13$ obtained by means of a micromachined oscillator which are consistent with (44) but exclude (3). Here we also discuss the possibility of using the fitting procedures in theory-experiment comparison and the recently proposed new model of patch potentials. ${ }^{14}$ Section 4 contains new information obtained from experiments with semiconductor $15-17$ and dielectric $19 \mid 20$ test bodies. These experiments are consistent with the dielectric permittivity (1) but exclude (2). The critical analysis of claimed observation of the thermal Casimir force ${ }^{21}$ is presented in Sec. 5. Here we show that the long-separation data of this experiment better agrees not with (3), as claimed, but with the dielectric permittivity (4). In Sec. 6 the reader will find our conclusions. 


\section{Is the PFA Exact Enough for Theory-Experiment Comparison?}

All precise measurements of the Casimir force performed to date use the configuration of a sphere (or a spherical lens) above a plate. To calculate the theoretical Casimir force $F$ in sphere-plate geometry one first calculates the free energy $\mathcal{F}$ between two parallel plates and then uses the PFA in the form $[5$

$$
F(d, T)=2 \pi R \mathcal{F}(d, T),
$$

where $d$ is the shortest separation between the sphere and the plate and between two plates, $R$ is the radius of the sphere. The error introduced from the use of the PFA can be estimated from the comparison with exact results for sphere-plate geometry. Recently it was shown $22 \mid 23$ that at $T=0$ the first two leading terms for the electromagnetic Casimir energy in the configuration of an ideal metal sphere above an ideal metal plate are given by

$$
E(d)=-\frac{\pi^{3} R \hbar c}{720 d^{2}}\left[1+\left(\frac{1}{3}-\frac{20}{\pi^{2}}\right) \frac{d}{R}\right]=-\frac{\pi^{3} R \hbar c}{720 d^{2}}\left(1-1.69 \frac{d}{R}\right) .
$$

For the force between a sphere and a plate from (6) one finds

$$
F(d)=-\frac{\pi^{3} R \hbar c}{360 d^{3}}\left[1+\left(\frac{1}{6}-\frac{10}{\pi^{2}}\right) \frac{d}{R}\right]=-\frac{\pi^{3} R \hbar c}{360 d^{3}}\left(1-0.85 \frac{d}{R}\right) .
$$

Thus, for a force, the leading correction to the PFA result is equal to $-0.85 d / R$. This is really a small correction if to take into consideration that the experimental range of parameters is given by

$$
0.001 \leq \frac{d}{R} \leq 0.007
$$

For an ideal metal sphere above an ideal metal plane it was also proven 24 that the PFA gives the same values of the Casimir force at $T=0$ and of the thermal correction to it as does the exact theory in the zeroth order of $d / R$ [with exception of only extremely low $\left.T \ll \hbar c /\left(2 k_{B} R\right)\right]$. Thus, for ideal metals the use of the PFA in sphere-plate geometry may result in only small errors less than $d / R$.

For a sphere and a plate made of metals described by the Drude and plasma models the exact computations of the Casimir force were performed $25 \mid 26$ for $d / R>$ 0.1. Deviations between the predictions of the PFA and the exact theory were described in terms of the quantity $q=F_{p} / F_{D}$, where $F_{p}$ and $F_{D}$ are the Casimir forces calculated using the simple plasma and Drude models, respectively, i.e., using Eqs. (3) and (4) with $\varepsilon_{c}(\omega)=1$. It was shown that deviations between the values of $q$ computed using the exact theory and the PFA decrease from $9.2 \%$ to $2.5 \%$ when $d / R$ decreases from 5 to 0.1 .

In the framework of the PFA in the high-temperature regime $T \gg T_{\text {eff }}=$ $\hbar c /\left(2 d k_{B}\right)$ or, alternatively, at large separations $d \gg \hbar c /\left(2 k_{B} T\right)$ it holds $q=2$ because under these conditions $\mathcal{F}_{p}(d, T)=2 \mathcal{F}_{D}(d, T)$. It was claimed, however, that at large separations the exact theory leads to $q=3 / 2$ instead of a factor $q=2$ as follows from the PFA. 25/26 This statement formulated in so general form 
is somewhat misleading because it does not take into consideration the application region of the PFA. In fact the value of $q$ depends on the interplay between different parameters. Thus, it really holds that

$$
q=\frac{3}{2} \quad \text { for } \quad \frac{2 \pi c}{\omega_{p}} \ll R \ll d .
$$

This is the limit of extremely large separations (much larger than the sphere radius). The condition (9) is outside the experimental region (8) and outside the application region of the PFA. Because of this it is not reasonable to compare the result $q=3 / 2$ with $q=2$, as obtained from the PFA.

There is also another case of large separations outside the application region of the PFA where one has 27

$$
q=1 \quad \text { for } \quad d \gg R, \quad d \gg \frac{\hbar c}{2 k_{B} T}, \quad R \leq \frac{2 \pi c}{\omega_{p}} .
$$

Finally, exact computations lead 28 to the same result as the PFA

$$
q=2 \text { for } \quad \frac{\hbar c}{2 k_{B} T} \ll d \ll R .
$$

This is just the region of large separations which simultaneously belongs to the application region of the PFA. For instance, exact computations show $25 \mid 26$ that at $d=5 \mu \mathrm{m} q$ increases from approximately 1.48 to 1.63 when $d / R$ decreases from 2.5 to 0.5 . With further decreasing of $d / R$ the quantity $q$ goes to 2 , as follows from the PFA.

One can conclude that in the region of the experimental parameters (8) the PFA is well applicable for the comparison between experiment and theory in the configuration of a perfectly shaped sphere (spherical lens) above a plane plate.

\section{Experiments Between Metallic Test Bodies Using a Micromachined Oscillator}

In several successive experiments $12|13| 29 \mid 30$ the Casimir pressure between two $\mathrm{Au}$ plates was determined from dynamic measurements of the gradient of the Casimir force betwen a sphere and a plate. These experiments were performed in the separation region $160 \mathrm{~nm} \leq d \leq 750 \mathrm{~nm}$. Their results gave rise to continuing hot discussions because contrary to expectations they were found to exclude the dielectric permittivity of the Drude model and to be consistent with the dielectric permittivity of the plasma model. Here we present the comparison of the measurement data with theory $12[13$ in the region of the largest separations $700 \mathrm{~nm} \leq d \leq 750 \mathrm{~nm}$ overlapping with another recent experiment ${ }^{21}$ leading to the opposite conclusions (see Sec. 5).

In Fig. 1(a,b) the mean measured Casimir presures are shown as crosses. The arms of the crosses indicate the total experimental errors in separations and pressures determined (a) at a $95 \%$ confidence level and (b) at a $67 \%$ confidence level. 

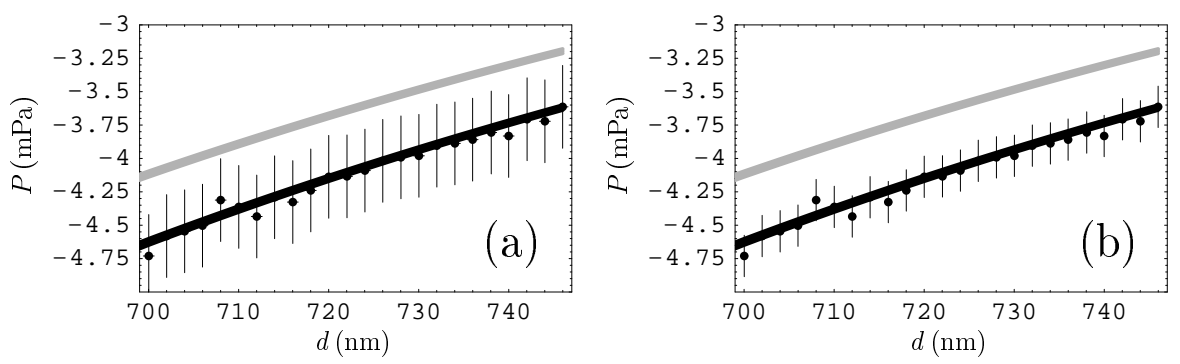

Fig. 1. The experimental data for the Casimir pressure between two parallel plates measured by means of micromechanical torsional oscillator as a function of separation are shown as crosses. The arms of the crosses indicate the total experimental errors determined at (a) $95 \%$ and (b) $67 \%$ confidence level. The grey and black lines show the theoretical Casimir pressures computed using the Drude and plasma model approaches, respectively. The thickness of the lines indicates the total theoretical errors.

The upper (grey) and lower (black) lines show theoretical Casimir pressures computed using the Drude and plasma model approaches. Note that the Drude model approach does not reduce to the use of dielectric permittivity (3). It exploits the tabulated optical data 31 extrapolated to lower frequencies by means of the simple Drude model. The point is that (3) does not provide a sufficiently accurate picture for the optical data of $\mathrm{Au}$ in the frequency region below the first absorbtion band. To model the optical data in that region using Eq. (3) one should consider $\frac{32}{12}$ the frequency-dependent relaxation parameter $\gamma(\omega, T)$. The experimental results $12 \mid 13$ exclude the predictions of the Drude model approach over the entire measurement range from 162 to $746 \mathrm{~nm}$. Figure 1(a,b) convincingly demonstrates this fact at $d>700 \mathrm{~nm}$. Possible theoretical reasons why the experimental data $12 \mid 13$ are consistent with the plasma model, which does not include dissipation, and exclude a more correct Drude model are discussed in Ref. 33.

\subsection{What is changed if one uses the fitting procedure between the data and the theoretical Casimir force}

The comparison between experiment and theory shown in Fig. 1(a,b) is independent in the sense that the measured Casimir forces were not used to specify any theoretical parameter. Let us now admit that some parameter of the theoretical Casimir force is unknown. The question arises whether it is possible to determine it by fitting the measurement data to theory and simultaneously choose between two different theoretical approaches. To answer this question, we perform the $\chi^{2}$-fit of the data in Fig. 1(a,b) to the perturbation expansions of the Lifshitz formula for the Casimir pressure using the simple plasma and Drude models ${ }^{[5}$ Such expansions are well applicable at $d>700 \mathrm{~nm}$. Thus, for the plasma model one has 5

$$
P_{p}^{\mathrm{th}}(d, \delta)=-\frac{\pi^{2} \hbar c}{240 d^{4}}\left(1-\frac{16}{3} \frac{\delta}{d}+24 \frac{\delta^{2}}{d^{2}}\right),
$$


where $\delta=\lambda_{p} /(2 \pi)=c / \omega_{p}$ is the penetration depth of electromagnetic fluctuations into the metal.

We now assume for a moment that the value of $\delta$ for $\mathrm{Au}$ is not known and find it from the best fit between $P_{p}^{\text {th }}$ and 24 experimental data points $P_{i}$ in Fig. 1(b). The minimization of the $\chi^{2}$-function

$$
\chi^{2}=\sum_{i-1}^{N} \frac{\left|P_{i}-P^{\mathrm{th}}\left(d_{i}, \delta_{1}, \ldots, \delta_{k}\right)\right|}{\left(\Delta P_{i}\right)^{2}},
$$

where $N=24, \Delta P_{i}$ are the total experimental errors determined at a $67 \%$ confidence level, $P^{\text {th }}=P_{p}^{\text {th }}, \delta_{1}=\delta$ and $k=1$ leads to $\chi_{\min }^{2}=1.88$ and $\delta=21.25 \mathrm{~nm}$. This result is in a very good agreement with the independently known value $\delta^{(\mathrm{Au})}=$ $22 \mathrm{~nm}$. The value of the $\chi^{2}$-probability $P\left(\chi^{2}>\chi_{\min }^{2}\right)$, i.e., the probability of an event that in a new measurement the value of $\chi^{2}$ larger than $\chi_{\min }^{2}$ will be obtained, can be found using the number of degrees of freedom $f=N-k=23$. The result is $P\left(\chi^{2}>\chi_{\min }^{2}\right)=0.9999$ which confirms that the plasma model is in a very good agreement with the data. This conclusion was obtained from the fit to the mean measured Casimir pressures. The same fit repeated with each of 33 sets of individual measurements results in

$$
0.07 \leq P\left(\chi^{2}>\chi_{\min }^{2}\right) \leq 0.9, \quad 17 \mathrm{~nm} \leq \delta \leq 24 \mathrm{~nm} .
$$

Now we repeat the fitting procedure but use the theoretical pressures obtained from the Drude model approach $\frac{5}{5}$

$$
P_{D}^{\mathrm{Th}}(d, \delta)=-\frac{\pi^{2} \hbar c}{240 d^{4}}\left(1-\frac{16}{3} \frac{\delta}{d}+24 \frac{\delta^{2}}{d^{2}}\right)+\frac{k_{B} T \zeta(3)}{8 \pi d^{3}}\left(1-6 \frac{\delta}{d}+24 \frac{\delta^{2}}{d^{2}}\right) .
$$

From the mean measured Casimir pressures in Fig. 1(b) one obtains

$$
\chi_{\min }^{2}=2.42, \quad P\left(\chi^{2}>\chi_{\min }^{2}\right)=0.9999, \quad \delta=4.2 \mathrm{~nm} .
$$

This could be also considered as a very good agreement with the data if the correct value of $\delta^{(\mathrm{Au})}=22 \mathrm{~nm}$ were not independently known. The fit to different individual sets of measurements leads to

$$
0.07 \leq P\left(\chi^{2}>\chi_{\min }^{2}\right) \leq 0.9, \quad 2.5 \mathrm{~nm} \leq \delta \leq 6 \mathrm{~nm} .
$$

Keeping in mind that all the values of $\delta$ obtained from the fit to the Drude model Casimir pressure (15) are in complete disagreement with the correct value, the theoretical description of the data by means of Eq. (15) should be rejected in spite of a high $\chi^{2}$-probability.

From the above one can conclude that the fit of the experimental data to two competing theoretical approaches containing unknown parameters may not allow for a reliable choice between these approaches. 


\subsection{Could the patch effect compensate differences between the experimental data and the Drude model prediction?}

It is common knowledge that the patch potentials due to grain structure of $\mathrm{Au}$ coatings, surface contaminants etc. lead to additional electric force which should be taken into account in precision measurements. Using the earlier proposed model of patches 34 it was shown $\frac{30}{30}$ that they lead to a negligibly small contribution to the Casimir pressure. Recently the so-called quasi-local model of patches was suggested 14 which leads to the orders of magnitude larger electric pressure than that predicted in Ref. 34. In this connection it was claimed 14 that "patches may render the experimental data at distances below 1 micrometer compartible with theoretical predictions based on the Drude model". In support of this claim a fit of the suggested patch model to the difference between the measurement results 12 and the theoretical prediction for the Casimir pressure based on the Drude model (called the residual signal) was performed. It was found that for the maximum patch size approximately equal to $l_{\max }=1074 \mathrm{~nm}$ and the root-mean-square voltage of $V_{r m s}=12.9 \mathrm{mV}$ there is a qualitative agreement between the residual signal and the fitted patch pressure to within a few percent of the total measured pressure. Such large patches cannot be connected with the grain structure of the surface and were attributed to some hypothetical contaminants 14

With respect to this result it should be stressed that the theoretical pressures predicted by the Drude model were calculated 14 not as in Refs. 12, 13 (i.e., using the tabulated optical data 31 extrapolated to low frequencies using the simple Drude model) but with the help of Eq. (3) with three oscillators. As was noted above, this leads to significant deviations in the dielectric permittivity and therefore in the computational results for the Casimir pressure. Moreover, the surface roughness was not taken into account in Ref. 13. For comparison purposes in Fig. 2 we plot the magnitudes of the residual signal as a function of separation for the Drude model theoretical prediction as in Ref. 14 (the upper set of crosses shown at a $67 \%$ confidence level) and as in Refs. 12, 13 (the lower set of crosses shown at the same confidence level). As can be seen in Fig. 2, at separation distances below $d=300 \mathrm{~nm}$ the residual signal used in Ref. 14 deviates significantly from a more precise result $12[13$

It is worthy of note also that the agreement between the residual signal and the patch pressure is up to a few percent of the total measured pressure 14 This should be compared with the relative differences between the theoretical predictions using the Drude and plasma model approaches which are also of about a few percent of the total measured pressures at separations below $1 \mu \mathrm{m}$. Thus, the statement made reduces to saying that the patch effect and the residual signal are of the same order of magnitude.

To check whether the suggested patch model can be employed to explain the residual signal $\left|P^{\text {expt }}-P_{D}^{\text {th }}\right|$, we have calculated the value of $\chi_{\min }^{2}$ using Eq. (13) with $P^{\text {th }}=P_{D}^{\text {th }}$, as computed in Ref. 14, and two fitting parameters $\delta_{1}=l_{\max }$ 


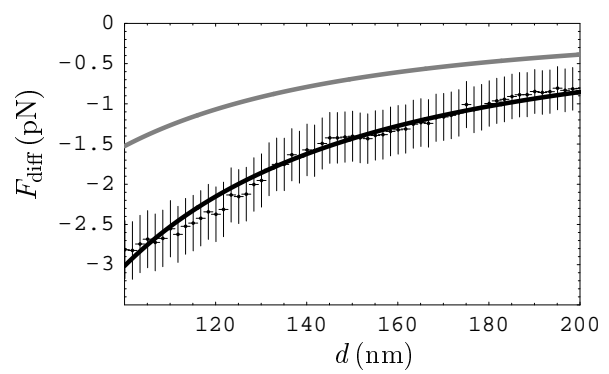

Fig. 2. The experimental data for the difference Casimir force between an Au sphere and Si plate measured by means of AFM as a function of separation are shown as crosses. The arms of the crosses indicate the total experimental errors determined at a $95 \%$ confidence level. The black and grey lines show the theoretical difference forces computed with neglected and included dc conductivity of Si plate in the absence of light, respectively.

and $\delta_{2}=V_{r m s}$ specified above. It was found that $\chi_{\min }^{2} \approx 700$. Taking into account that the number of degrees of freedom is equal to $f=291$, from this we obtain that $P\left(\chi^{2}>\chi_{\min }^{2}\right)=0$ with at least 8 zeros after a decimal comma. This result means that the theoretical pressure-distance dependence caused by the quasi-local patches $\frac{14}{\text { is irrelevant to the difference between the experimental data } 1213}$ and the predictions of the Lifshitz theory combined with the Drude model.

In spite of the above result obtained with respect to the quasi-local model of patches, it would be interesting to perform direct measurements of patch distributions on specially cleaned and prepared surfaces in high vacuum, as are used in measurements of the Casimir force. This would bring reliable model-independent information concerning the role of patch potentials in Casimir physics.

\section{Experiments with Semiconductor and Dielectric Test Bodies}

Here we briefly review several recent experiments which demonstrate that the Lifshitz theory with taken into account dc conductivity of dielectric materials is excluded by the measurement data.

\subsection{Optical modulation of the Casimir force between an Au sphere and Si plate}

In this experiment $\frac{15[16}{\text { an }}$ Au sphere was attached to the cantilever of the atomic force microscope above a Si plate illuminated with laser pulses. The measured quantity was the difference in the Casimir forces between a sphere and a plate in the presence and in the absence of laser light on the plate. In the absence of light $\mathrm{Si}$ was in a dielectric state with the density of charge carriers of about $n=5 \times 10^{14} \mathrm{~cm}^{-3}$. In the presence of laser pulse the density of charge carriers was higher up to 5 orders of magnitude. Thus, in the presence of light $\mathrm{Si}$ was in metallic state with $n>n_{c r}$, where $n_{c r}$ is the critical density of charge carriers such that the phase transition from dielectric to metallic state occurs. 
In Fig. 3 we demonstrate as crosses the typical measured differences in the Casimir forces, $F_{\text {diff }}$, versus separation between the sphere and the plate. The arms of the crosses indicate the total experimental errors determined at a $95 \%$ confidence level. The grey line shows the results of computations using the Lifshitz theory with included contribution of free charge carriers, both in the absence and in the presence of laser light on a Si plate. As can be seen in Fig. 3, this theoretical approach is excluded by the experimental data at a $95 \%$ confidence level. The black line shows the theoretical results obtained with included contribution of free charge carriers in the presence of laser light (i.e., when $\mathrm{Si}$ was in metallic state), but with charge carriers disregarded in the absence of laser light on a Si plate (i.e., when $\mathrm{Si}$ was in dielectric state). From Fig. 3 it is seen that the black line is fully consistent with the experimental data. Thus, the inclusion of dc conductivity of dielectric $\mathrm{Si}$ in the Lifshitz theory is in contradiction to the optical modulation experiment.

\subsection{The Casimir force between an $A u$ sphere and an indium tin oxide plate}

In this experiment $\frac{17 / 18}{16}$ the atomic force microscope was used to measure the Casimir force between an $\mathrm{Au}$ sphere and an indium tin oxide (ITO) plate as a function of separation (for the details of calibration of a setup see Ref. 35). Then the ITO plate was UV-treated and measurements of the Casimir force were repeated. The experimental results for the untreated ITO sample are shown as the lower set of crosses in Fig. 4(a). These results are consistent with earlier measurements of the Casimir force gradient between an Au sphere and an ITO plate. $\underline{36}$ [37 The measurement data for the untreated sample are in good agreement with theoretical results computed using the Lifshitz theory with included contribution of free charge carriers (the lower pair of solid lines forms the theoretical band; the thickness of this band is caused by a freedom in the extrapolation of the measured optical data of ITO to higher frequencies).

Quite unexpectedly, the measured Casimir force from the UV-treated sample was found to differ significantly from the untreated one. It is shown as the upper set of crosses in Fig. 4(a). In fact the decrease in the force magnitude ranging from $21 \%$ to $35 \%$ depending on separation was observed. The same set of crosses demonstrating the Casimir force from the UV-treated sample is shown in Fig. 4(b). The optical data for a UV-treated sample were measured and found almost the same as for an untreated one. This is in conflict with the fact that the magnitudes of the Casimir force obtained after a UV treatment are much smaller than for an untreated sample. The computational results for the UV-treated sample with included contribution of ITO charge carriers are shown by the pair of dashed lines in Fig. 4(b). The computational results with disregarded contribution of ITO charge carriers are shown by the pair of upper solid lines in Fig. 4(a). As can be seen in Fig. $4(a, b)$, the inclusion of free charge carriers for the UV-treated ITO sample results in complete disagreement between the data and the theory, whereas the 

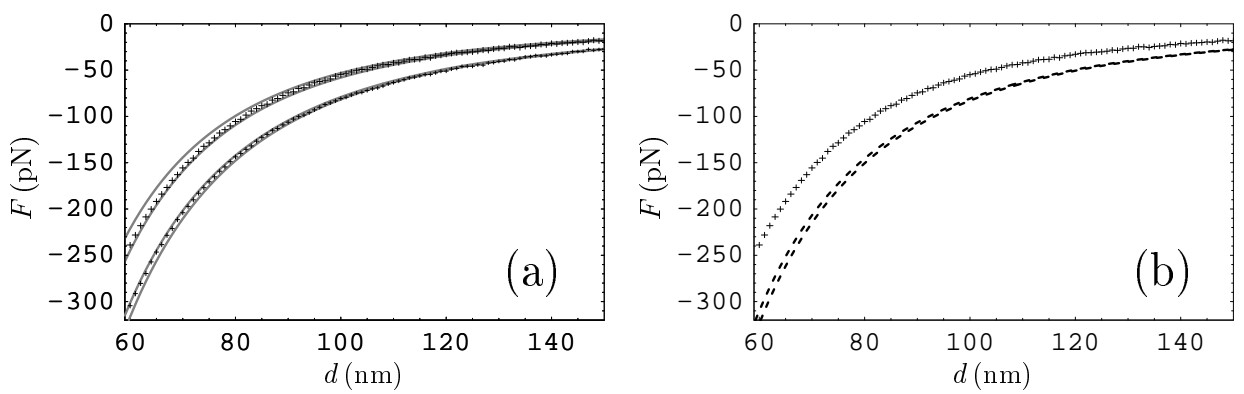

Fig. 3. (a) The mean measured Casimir force as function of separation is indicated as crosses for the untreated (the lower set of crosses) and UV-treated (the upper set of crosses) sample. The theoretical Casimir force shown by the pairs of solid lines for the untreated sample is calculated with free charge carriers included and for the UV-treated sample with free charge carriers omitted. (b) The mean measured Casimir force as a function of separation (crosses) and the theoretical results computed with included contribution of free charge carriers (two dashed lines) for the UV-treated sample.

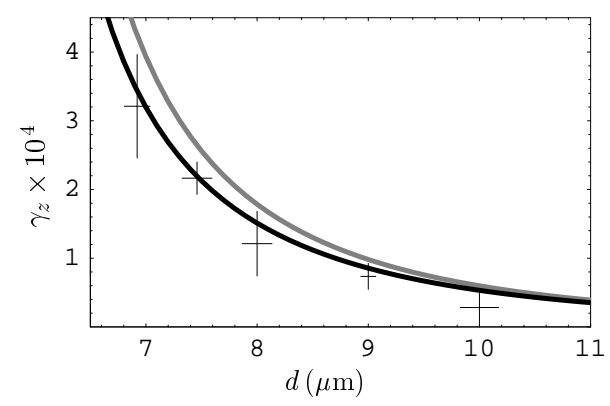

Fig. 4. The experimental data for the fractional change of the trap frequency due to the CasimirPolder force between ${ }^{87} \mathrm{Rb}$ atoms and fused silica plate are indicated by crosses as a function of separation. The total experimental errors are determined at a $70 \%$ confidence level. The black and grey lines show the theoretical fractional shift computed with neglected and included dc conductivity of fused silica.

neglect of free charge carriers makes theory consistent with the measurement data. It was suggested 17 that the UV treatment of ITO causes the phase transition from metallic to dielectric state (this is confirmed by the fact ${ }^{38}$ that the UV treatment leads to lower mobility of charge carriers). If this is the case, than the neglect of free charge carriers in the UV-treated ITO sample can be compared with the neglect of dc conductivity in dielectric Si considered above ${ }^{39}$ It remains unexplained, however, why one should disregard the dc conductivity of a dielectric body when calculating the Casimir force in the framework of the Lifshitz theory. 


\subsection{Frequency shift of center-of-mass oscillations due to the Casimir-Polder force}

The Casimir-Polder force between an atom and a plate leads to a fractional shift $\gamma_{z}$ of the center-of-mass oscillation frequency $\omega_{z}$ of the Boze-Einstein condensate in the direction perpendicular to the plate. This shift was measured 19 for the condensate of ${ }^{87} \mathrm{Rb}$ atoms and $\mathrm{SiO}_{2}$ dielectric plate at separations from 7 to $11 \mu \mathrm{m}$, i.e., in the region of thermal Casimir-Polder force, both at thermal equilibrium and out of equilibrium when the temperature of a plate was higher than that of environment. The results obtained were compared with the Lifshitz theory and its generalization for a nonequilibrium situation. As an example, in Fig. 5 the experimental results for $\gamma_{z}$ as a function of separation are shown as crosses for the plate at $T=605 \mathrm{~K}$ and an environment at $T=310 \mathrm{~K}$. The total experimental errors in both $\gamma_{z}$ and $d$ are indicated at a $67 \%$ confidence level. The black solid line shows the theoretical results obtained 19 with disregarded dc conductivity of $\mathrm{SiO}_{2}$. These results are consistent with the measured data. The grey line in Fig. 5 shows the theoretical results computed 20 with dc conductivity of $\mathrm{SiO}_{2}$ included. As can be seen in Fig. 5, the theory with taking into account dc conductivity of dielectric $\mathrm{SiO}_{2}$ is excluded by the data. This again confirms the phenomenological prescription 516 that in computations of the Casimir force the contribution of free charge carriers of dielectrics and semiconductors of dielectric type should be disregarded. Needless to say that such kind phenomenological prescriptions cannot be considered as conclusive explanations and need justification on the basis of first principles.

\section{Torsion Balance Experiments}

Contrary to the previous experiments which used spheres of $100-150 \mu \mathrm{m}$ radii or even atoms to measure the Casimir (Casimir-Polder) force, torsion balance experiments exploit spherical lenses with $15-20 \mathrm{~cm}$ radii of curvature. The use of large lenses allows to significantly increase the Casimir force but makes problematic sufficiently precise characterization of the surface. Below we consider two recent experiments employing large spherical lenses to measure the Casimir force which arrive to the conflicting conclusions.

\subsection{Confirmation of the thermal Casimir force predicted by the plasma model}

The first 40 of two recent torsion balance experiments used a lens of $R=20.7 \mathrm{~cm}$ radius of curvature and a plate both coated with $\mathrm{Au}$. The measured data for the force gradient over the separation region from 0.48 to $6.5 \mu \mathrm{m}$ were fitted to the sum of contributions from the residual electric force and from the theory of the thermal Casimir force based on the plasma model. The roughness corrections were also taken into account. The fit used Eq. (13) with one fitting parameter (the residual potential difference $\left.V_{\text {res }}\right)$. 
As a result, the minimum value of the $\chi^{2}$-function was found $\chi_{\min }^{2}=513$ with $V_{\text {res }}=20.0 \pm 0.2 \mathrm{mV}$. Taking into account that the number of degrees of freedom was $f=558$, from this one obtains $P\left(\chi^{2}>\chi_{\min }^{2}\right)=0.91$. This demonstrates the high level of agreement between the data and the used theory of the Casimir force and supports the results of Refs. 12, 13, 29] and 30 considered in Sec. 3.

\subsection{Claimed observation of the thermal Casimir force predicted by the Drude model}

The second 21 of two recent torsion balance experiments used a lens of $R=15.6 \mathrm{~cm}$ radius of curvature. Both the lens and the plate were coated with $\mathrm{Au}$. The measured data for the total force $F_{\text {tot }}(d)$ over the separation region from 0.7 to $7.3 \mu$ m were fitted to the sum of the Casimir force, the hypothetical electrostatic force due to large patches, and some constant contribution $-a$ called an offset due to voltage offsets in the measurement electronics. 21

The Casimir force $F(d)$ was calculated in the framework of both the Drude and the plasma model approaches. The form of the force due to large patches remained unknown. According to the authors, 21 "an independnet measurement of this electrostatic force with the required accuracy is currently not feasible." It was suggested that there are large patches of size $\lambda$ on Au-coated surfaces such that

$$
d \ll \lambda \ll \sqrt{R d}
$$

due to absorbed impurities or oxides. The electric force due to these patches was modeled by the term 21

$$
F_{\text {patch }}(d)=-\pi \epsilon_{0} R \frac{V_{\mathrm{rms}}^{2}}{d},
$$

where $\epsilon_{0}$ is the permittivity of the free space, and $V_{\mathrm{rms}}$ is the magnitude of voltage fluctuations across the text bodies. In view of this, after the application of voltage in order to cancel the electric force due to residual potential difference, the total measured force was represented in the form

$$
F_{\text {tot }}(d)=F(d)-\pi \epsilon_{0} R \frac{V_{\mathrm{rms}}^{2}}{d}-a .
$$

As a next step, Ref. 21 performed the $\chi^{2}$ fit of the mean data for the total force measured at 21 separation distances to Eq. (20) with the two fitting parameters $V_{\mathrm{rms}}$ and $a$. Note that the experimental errors indicated 21 in the mean total forces do not include the systematic constituents and are unreasonably small. For example, at the largest separation, $d=7.29 \mu \mathrm{m}$, the measured mean total force is equal to $F_{\text {tot }}=(19.54 \pm 0.28) \mathrm{pN}$, i.e., the relative error is equal to only $1.4 \%$ (see Ref. 41 for a more detailed discussion of the errors in this experiment). Here we should only stress that inclusion of the systematic errors into the fitting procedure seems necessary.

When the Drude model approach to the Casimir force is used, from the fit performed it was obtained 21 that $\chi_{\min }^{2}=19.76$ with the fitting parameters $a=$ 
$-3.0 \mathrm{pN}$ and $V_{\mathrm{rms}}=5.4 \mathrm{mV}$. This was characterized as an excellent agreement with the data. Taking into account, however, that the number of degrees of freedom $f=19$ one obtains that $P\left(\chi^{2}>\chi_{\min }^{2}\right)=0.41$. Such a value could be considered as being in favor of the model used if the results of an individual measurement were fitted. Here, however, the mean measured total force averaged over a large number of repetitions was used in the fit. In that case the $\chi^{2}$-probability should be larger than at least $50 \%$ in order to measured data could be considered as supporting the theoretcal model. The plasma model approach was excluded ${ }^{21}$ basing on the following results of the fit: $\chi_{\min }^{2}=608$ and $V_{\mathrm{rms}}=5.4 \mathrm{mV}$.

There is, however, one more weak point in the results of Ref. 21. The problem is that surfaces of lenses with centimeter-size radii of curvature have local deviations from perfect sphericity such as bubbles, pits and scratches. The impact of such deviations on the Casimir force was examined in detail.42 Specifically, it was shown that if a surface defect is located near the point of closest approach to the plate the simplest formulation of the PFA in Eq. (5) used in Ref. 21 is not applicable and should be replaced by more sophisticated equations. The characteristic lateral sizes of invariably present surface defects on mechanically polished and ground glass surfaces allowed by the optical surface specification data may vary from a few micrometers to a millimeter. In so doing their sizes in the normal direction to the surface are below a fraction of micrometer and their local radii of curvature may differ by tens of percent from the lens radius $R .42[43$ This is not in contradiction with the fact that the value of $R=15.6 \mathrm{~cm}$ was measured with the interferometric microscope and found to vary by less than $2 \%$ over the surface of the lens. ${ }^{21}$ The point is that interferometric microscopy does not provide values of the lens radius of curvature at separate points, but averaged values over about $0.5 \mathrm{~mm}$ regions. It was shown $\frac{42 \mid 43}{4}$ that at $d<3 \mu \mathrm{m}$ local surface defects could contribute significantly in the analysis and simulate differences between the predictions of the Drude and plasma model approaches.

Because of this it was suggested 141 temporarily disregard all the experimental data 21 at $d<3 \mu \mathrm{m}$ and repeat the analysis at $d>3 \mu \mathrm{m}$ where the influence of surface defects on the Casimir force is negligibly small. In this case the best agreement between the data and Eq. (20) computed using the Drude model approach is achieved with $\chi_{\min }^{2}=6.6, a=-0.29 \mathrm{pN}$ and $V_{\mathrm{rms}}=5.45 \mathrm{mV}$. Taking into account that $f=4$, we arrive at $P\left(\chi^{2}>\chi_{\min }^{2}\right)=0.16$. This demonstrates a poor agreement of the data with the Drude model.

In the case of the plasma model approach the best agreement between the data and Eq. (20) is achieved with $\chi_{\min }^{2}=2.68, a=3.6 \mathrm{pN}$ and $V_{\mathrm{rms}}=4.5 \mathrm{mV}$. With $f=4$ this results in $P\left(\chi^{2}>\chi_{\min }^{2}\right)=0.67$, i.e. the plasma model approach is in a very good agreement with the data at $d>3 \mu \mathrm{m}$. This is illustrated in Fig. 6 where the experimental data for the magnitudes of the total force multiplied by $d$ are shown as crosses and the predictions of the plasma and Drude model approaches are given as the black and grey lines, respectively. 


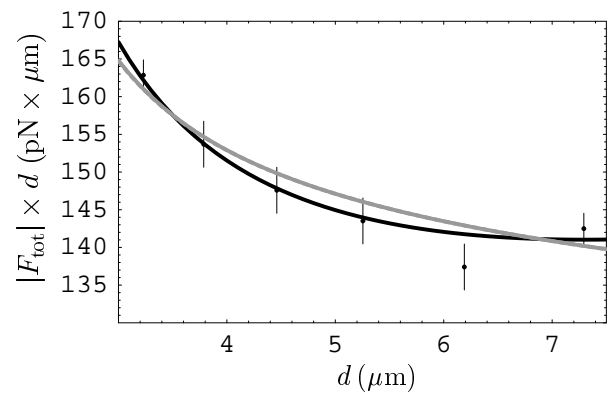

Fig. 5. The experimental data for the magnitudes of the mean measured force multiplied by separation are shown as crosses. The arms of the crosses indicate the experimental errors. The grey and black lines demonstrate the best fit of the total theoretical force to the experimental data computed using the Drude and plasma models, respectively, with two fitting parameters.

\section{Conclusions}

From the foregoing one arrives at the following conclusions.

The comparison of the PFA with the exact results for a sphere above a plate show that the PFA is well applicable to all performed experiments in sphere-plate geometry.

A number of experiments demonstrate that the inclusion of relaxation of free charge carriers for metals and dc conductivity for dielectrics in the Lifshitz theory leads to contradictions with the measurement data. This fact awaits for a complete theoretical explanation.

The comparison between a fitting procedure and an independent theoretical calculation where it is available shows that the fit by itself cannot be used to make a choice between two competing theoretical approaches.

The suggested quasi-local model of patches is incapable to explain the difference between the results of experiment using a micromachined oscillator and the Drude model approach to the Casimir force.

The claimed observation of the thermal Casimir force, as predicted by the Drude model approach, is not an independent measurement, but a fit using two fitting parameters. At separations above $3 \mu \mathrm{m}$ the data of this experiment are in agreement not with the Drude, but with the plasma model. Below $3 \mu \mathrm{m}$ a seeming agreement with the Drude model can be explained by disregard of surface imperfections.

\section{Acknowledgments}

G.L.K. and V.M.M. were partially supported by the NSF Grant No. PHY0970161 and by DFG grant BO 1112/20-1.

\section{References}

1. H. B. G. Casimir, Proc. K. Ned. Akad. Wet. B 51, 793 (1948).

2. M. Bordag, U. Mohideen and V. M. Mostepanenko, Phys. Rep. 353, 1 (2001). 
3. K. A. Milton, J. Phys. A 37, R209 (2004).

4. S. K. Lamoreaux, Rep. Progr. Phys. 68, 201 (2005).

5. M. Bordag, G. L. Klimchitskaya, U. Mohideen and V. M. Mostepanenko, Advances in the Casimir Effect (Oxford University Press, Oxford, 2009).

6. G. L. Klimchitskaya, U. Mohideen and V. M. Mostepanenko, Rev. Mod. Phys. 81, $1827(2009)$.

7. A. W. Rodriguez, F. Capasso and S. G. Johnson, Nature Photon. 5, 211 (2011).

8. G. L. Klimchitskaya, U. Mohideen and V. M. Mostepanenko, Int. J. Mod. Phys. B 25, 171 (2011).

9. V. B. Bezerra, G. L. Klimchitskaya, V. M. Mostepanenko and C. Romero, Phys. Rev. A 69, 022119 (2004).

10. B. Geyer, G. L. Klimchitskaya and V. M. Mostepanenko, Phys. Rev. D 72, 085009 (2005).

11. M. Bordag and I. G. Pirozhenko, Phys. Rev. D 82, 125016 (2010).

12. R. S. Decca, D. López, E. Fischbach, G. L. Klimchitskaya, D. E. Krause and V. M. Mostepanenko, Phys. Rev. D 75, 077101 (2007).

13. R. S. Decca, D. López, E. Fischbach, G. L. Klimchitskaya, D. E. Krause and V. M. Mostepanenko, Eur. Phys. J. C 51, 963 (2007).

14. R. O. Behunin, F. Intravaia, D. A. R. Dalvit, P. A. Maia Neto and S. Reynaud, Phys. Rev. A 85, 012504 (2012).

15. F. Chen, G. L. Klimchitskaya, V. M. Mostepanenko and U. Mohideen, Optics Express 15, 4823 (2007).

16. F. Chen, G. L. Klimchitskaya, V. M. Mostepanenko and U. Mohideen, Phys. Rev. B 76, 035338 (2007).

17. C.-C. Chang, A. A. Banishev, G. L. Klimchitskaya, V. M. Mostepanenko and U. Mohideen, Phys. Rev. Lett. 107, 090403 (2011).

18. A. A. Banishev, C.-C. Chang, R. Castillo-Garza, G. L. Klimchitskaya, V. M. Mostepanenko and U. Mohideen, Phys. Rev. B 85 (2012), to appear.

19. J. M. Obrecht, R. J. Wild, M. Antezza, L. P. Pitaevskii, S. Stringari and E. A. Cornell, Phys. Rev. Lett. 98, 063201 (2007).

20. G. L. Klimchitskaya and V. M. Mostepanenko, J. Phys. A: Math. Theor. 41, 312002 (2008).

21. A. O. Sushkov, W. J. Kim, D. A. R. Dalvit and S. K. Lamoreaux, Nature Phys. 7, 230 (2011).

22. G. Bimonte, T. Emig, R. L. Jaffe and M. Kardar, arXiv:1110.1082v1.

23. L. P. Teo, M. Bordag and V. Nikolaev, arXiv:1110.5100 v1.

24. M. Bordag and I. Pirozhenko, Phys. Rev. D 81, 085023 (2010).

25. A. Canaguier-Durand, P. A. Maia Neto, A. Lambrecht and S. Reynaud, Phys. Rev. Lett. 104, 040403 (2010).

26. A. Canaguier-Durand, P. A. Maia Neto, A. Lambrecht and S. Reynaud, Phys. Rev. A 82, 012511 (2010).

27. R. Zandi, T. Emig and U. Mohideen, Phys. Rev. B 81, 195423 (2010).

28. B. Geyer, G. L. Klimchitskaya and V. M. Mostepanenko, Phys. Rev. A 82, 032513 (2010).

29. R. S. Decca, E. Fischbach, G. L. Klimchitskaya, D. E. Krause, D. López and V. M. Mostepanenko, Phys. Rev. D 68, 116003 (2003).

30. R. S. Decca, D. López, E. Fischbach, G. L. Klimchitskaya, D. E. Krause and V. M. Mostepanenko, Ann. Phys. (N.Y.) 318, 37 (2005).

31. Handbook of Optical Constants of Solids, Vol. 1, ed. E. D. Palik (Academic, New York, 1985). 
32. I. M. Lifshitz, M. Ya. Azbel' and M. I. Kaganov, Electron Theory of Metals (Consultants Bureau, New York, 1973).

33. M. Bordag, Eur. Phys. J. C 71, 1788 (2011).

34. C. C. Speake and C. Trenkel, Phys. Rev. Lett. 90, 160403 (2003).

35. A. A. Banishev, C.-C. Chang and U. Mohideen, Int. J. Mod. Phys. A 26, 3900 (2011); Int. J. Mod. Phys.: Conf. Ser. 3, 497 (2011);

36. S. de Man, K. Heeck, R. J. Wijngaarden and D. Iannuzzi, Phys. Rev. Lett. 103, 040402 (2009).

37. S. de Man, K. Heeck and D. Iannuzzi, Phys. Rev. A 82, 062512 (2010).

38. C. N. Li, A. B. Djurišić, C. Y. Kwong, P. T. Lai, W. K. Chan and S. Y. Liu, Appl. Phys. A 80, 301 (2005).

39. U. Mohideen, A. A. Banishev, C.-C. Chang, R. Castillo-Garza, G. L. Klimchitskaya and V. M. Mostepanenko, Int. J. Mod. Phys. A, this issue.

40. M. Masuda and M. Sasaki, Phys. Rev. Lett. 102, 171101 (2009).

41. G. L. Klimchitskaya, M. Bordag, E. Fischbach, D. E. Krause and V. M. Mostepanenko, Int. J. Mod. Phys. A 26, 3918 (2011); Int. J. Mod. Phys.: Conf. Ser. 3, 515 (2011).

42. G. L. Klimchitskaya and V. M. Mostepanenko, Int. J. Mod. Phys. A 26, 3944 (2011); Int. J. Mod. Phys.: Conf. Ser. 3, 541 (2011).

43. V. B. Bezerra, G. L. Klimchitskaya, U. Mohideen, V. M. Mostepanenko and C. Romero, Phys. Rev. B 83, 075417 (2011). 